\title{
Los sonidos de la Prehistoria: Reflexiones en torno a las evidencias de prácticas musicales del paleolítico y el neolítico en Eurasia ${ }^{1}$
}

\section{Sounds of Prehistory: Reflections on the Evidence of Palaeolithic and Neolithic Musical Practices in Eurasia}

\author{
Raquel JiMÉNEZ PASALOdOS \\ Universidad de Valladolid/Universidad de Barcelona \\ raquel.jimenez@uva.es \\ http://orcid.org/0000-0001-9422-8302 \\ Ana María AlARCón JiMÉNEZ \\ Universidad de Barcelona \\ am.alarconjimenez@ub.es \\ https://orcid.org/0000-0001-7555-257X \\ Neemías Santos da Rosa \\ Universidad de Barcelona \\ n.santosdarosa@ub.edu \\ https://orcid.org/0000-0001-8800-146X \\ Margarita DíAZ-ANDREU \\ ICREA y Universidad de Barcelona \\ m.diaz-andreu@ub.edu \\ http://orcid.org/0000-0003-1043-2336
}

Fecha de recepción: 07/01/2021

Fecha de aceptación: 03/03/2021

\section{RESUMEN}

En este artículo presentamos una breve introducción historiográfica y metodológica de la arqueología musical y la arqueoacústica como subdisciplinas de la arqueología encargadas del estudio de las evidencias materiales de las prácticas musicales del pasado remoto, y realizamos

1 Este trabajo se enmarca en el proyecto de investigación de la ERC Advanced Grant Artsoundscapes con el título "The sound of special places: exploring rock art soundscapes and the sacred" (EC Grant Agreement 787842), cuya Investigadora Principal es Margarita Díaz-Andreu y, como tal, firma en última posición. 
un recorrido por los principales hallazgos arqueológicos relacionados con la música, el sonido y la acústica de los espacios desde el Paleolítico al Neolítico en Eurasia. Además, proponemos una serie de hallazgos como casos de estudio que van a permitir reflexionar en torno a la importancia cultural de la musicalidad, y los posibles usos y funciones de la música en aquellas sociedades cazadoras-recolectoras y de primeros agricultores.

Palabras clave: Arqueología Musical, Arqueoacústica

Topónimos: Eurasia

Periodo: Paleolítico y Neolítico

\section{ABSTRACT}

In this paper, we present a brief historiographical and methodological introduction to music archeology and archaeoacoustics, archaeological subdisciplines that study the material evidence of musical practices in the distant past. Moreover, we briefly present some of the main archaeological finds linked to music, sound and acoustics from the Paleolithic to the Neolithic periods in Eurasia. Finally, we propose a series of discoveries as case studies that will allow us to reflect on the cultural importance of musicality, and the possible uses and functions of music in those hunter-gatherer and early farmer societies.

Key words: Music Archaeology, Archaeoacoustics

Toponyms: Eurasia

Period: Palaeolithic and Neolithic

\section{INTRODUCCIÓN}

En los últimos años, la neurociencia, los estudios cognitivos y la psicología evolutiva están aportando nuevos datos que muestran lo que la etnomusicología había propuesto desde hacía décadas: la musicalidad es una capacidad exclusiva del ser humano, y el canto, la danza y la construcción de instrumentos son fenómenos universales, ya que, aunque estos elementos estén culturalmente definidos, todas las sociedades vivas conocidas tienen comportamientos comparables que se pueden reconocer como musicales ${ }^{2}$. Líneas de investigación relativamente recientes y eminentemente interdisciplinares como la arqueología musical y la arqueoacústica están aportando un creciente número de pruebas sobre la importancia del sonido en las culturas de la prehistoria y la antigüedad. En este artículo proponemos una breve introducción historiográfica y metodológica a ambas subdisciplinas y un escueto recorrido por los principales hallazgos arqueológicos sobre música, sonido y acústica del espacio de las sociedades cazadoras-recolectoras y primeros agricultores de Eurasia, que van a permitir reflexionar tanto en torno a la importancia cultural que estas concedieron a la música y el sonido como componentes esenciales de su experiencia vital.

2 La noción de música empleada en este trabajo parte de una definición etic que engloba una serie de comportamientos universales que pueden ser reconocidos en diversas culturas y comparables entre sí, aunque el término como tal no exista entre algunos grupos humanos o presente marcadas diferencias (Gourlay, 1984; List, 1971 y 1984). Para una visión muy reciente de este campo se puede acceder a los vídeos de una conferencia virtual coorganizada entre el Museo Arqueológico Nacional y el proyecto Artsoundscapes celebrada el 18 de noviembre de 2020 sobre la "Arqueología de los espacios sonoros" realizada con motivo de la Vitrina 0 del Museo Arqueológico Nacional. Ver http://www.man.es/man/actividades/congresos-y-reuniones/ congresos-anteriores/2020/20201118-paisajes-sonoros.html ). 


\section{LOS ESTUDIOS SOBRE LA DIMENSIÓN SONORA DE LA PREHISTORIA}

La arqueología musical y la arqueoacústica son dos subdisciplinas de la arqueología que permiten, con herramientas metodológicas diversas y variados intereses teóricos, una aproximación a la dimensión sonora de las culturas pasadas. La primera se ocupa principalmente del estudio de las evidencias materiales de instrumentos sonoros que se encuentran en el registro arqueológico, ya que entre los comportamientos humanos asociados con la musicalidad se encuentran aquellos que producen y utilizan cultura material $\mathrm{y}$, por tanto, pueden dejar evidencias y patrones que perduran a través del tiempo. Estas evidencias materiales, organológicas e iconográficas, constituyen las fuentes primarias de la arqueología musical. En general, la disciplina se ha interesado por todos aquellos artefactos productores de sonido (Lund, 1981): los instrumentos de música propiamente dichos y otros materiales que, o bien fueron diseñados con intencionalidad acústica, o bien fueron usados con ese fin. Por otro lado, la arqueoacústica (Scarre y Lawson, 2006), pone su foco de interés en el estudio de los paisajes sonoros y de las características acústicas de los lugares que fueron habitados y utilizados por los seres humanos del pasado, ya sean naturales o de origen antrópico, y que en ocasiones revelan la importancia utilitaria, ritual o simbólica de determinados sonidos y parámetros acústicos. Pese a la pretensión globalizadora inicial del término arqueoacústica, la gran especificidad del análisis de los objetos sonoros ha llevado a que los especialistas en música antigua se hayan mantenido fieles a la denominación de arqueología musical, con lo que el nombre de arqueoacústica ha quedado reservado para aquellos que se dedican al estudio de los espacios sonoros.

Si bien ambas aproximaciones parecen relativamente recientes, el interés por la organología arqueológica tiene un largo recorrido. En territorio europeo, los primeros instrumentos musicales prehistóricos encontrados que suscitaron la curiosidad de arqueólogos y eruditos fueron las trompas irlandesas de la Edad del Bronce (B.B., 1763: Lám. 20; Cooper Walker, 1786). Poco después, la avidez de conocimiento del nacionalismo decimonónico por las historias locales pre-romanas produjo un crecimiento exponencial de los trabajos arqueológicos y, con ellos, nuevos hallazgos de interés musical. Si fue en Escandinavia donde se sentó la base de la arqueología prehistórica (gracias al Sistema de las Tres Edades desarrollado por Christian J. Thomsen), éste fue además un territorio privilegiado para el surgimiento del interés por la música arqueológica debido al también temprano descubrimiento de un nuevo tipo de trompas de la Edad del Bronce en Dinamarca en 1794. De hecho, el mismo Thomsen dató los imponentes aerófonos en 1836 (Thomsen, [1836] 1848: 52) y, basándose en las sagas islandesas, los bautizó con el nombre de lurs. A finales del siglo XIX ya se habían encontrado unos veinticinco ejemplares en diversos países escandinavos (Lund, 2010). En el Reino Unido sorprende también la regularidad con la que aparecen en el siglo XIX referencias a instrumentos musicales arqueológicos en una de las primeras publicaciones periódicas sobre arqueología, el Journal of the British Archaeological Association (Lawson, 2010: 107-134). Igualmente, la prensa arqueológica en Irlanda recoge artículos de interés musical con temas variados (P., 1833; Ball, 1844: 135-136; MacAdam, 1860: 99-110) ${ }^{3}$. El primer objeto organológico datado en las primeras etapas de la prehistoria apareció en 1871 en la cueva de Gourdan, en Haute-Garonne, y fue encontrado por el arqueólogo francés Édouard Piette, quien interpretó un hueso con tres perforaciones como una posible flauta neolítica (Piette, 1874a; Clodoré-Tissot et al., 2009: 38-39), y, pocos meses después, valoró la posibilidad de que algunos tubos de hueso provenientes de cuevas del Pirineo, hoy en día datados en el Auriñaciense o Magdaleniense

3 A pesar del hallazgo temprano de un probable pabellón de carnyx en Deskford, Escocia, en 1816, no se identificó como un posible fragmento del instrumento hasta 1959 (Piggott, 1959). 
(Martí Oliver et al., 2001: 53; Dauvois, 2005: 225), fuesen utilizados a modo de flautas de pan (Piette, 1874b). Este florecimiento de hallazgos arqueológicos, a los que hay que añadir también los provenientes de la arqueología mediterránea y de Oriente Medio, propiciaron que algunos autores comenzaran a dar nombre a la disciplina, primero en Francia, bajo la denominación archéologie musicale (Daussoigne-Mehul, 1848; Arbaud, 1857) y, después en Escandinavia en 1880, cuando el musicólogo Carl Erik Södling empleara el término musikarkeologi, es decir, arqueología de la música, en su discurso ante la Sociedad Anticuaria Sueca (Lund, 2010: 191).

En las primeras décadas del siglo pasado, el estudio de los instrumentos musicales arqueológicos más antiguos se llevó a cabo a partir de metodologías e intereses teóricos muy variados, procedentes de distintas disciplinas como la musicología comparada, la arqueología, la filología y la historia antigua (Jiménez Pasalodos, 2020: 39-99). La buena disposición teórica tanto de la arqueología postprocesual como de la etnomusicología favorecieron el florecimiento, a finales de los 70 , de investigaciones de temática arqueológico-musical. Los primeros contactos internacionales fructificaron en una sesión en la conferencia anual del International Council of Traditional Music (ICTM) que tuvo lugar en Berkeley en 1977, dedicada a debatir sobre la necesidad de una colaboración entre la etnomusicología y la arqueología (Lund, 2010 y comunicación personal). El encuentro fraguó en la creación del primer grupo de estudio internacional ${ }^{4}$ dedicado a la arqueología musical, el Study Group for Music Archaeology, reconocido en 1983 por el ICTM, la principal organización académica que se ocupa del estudio de las músicas no occidentales y de tradición oral. En las décadas de 1980 y 1990 aumentó significativamente el volumen de producción académica, incremento en parte animado por dicho grupo de estudios gracias a los congresos y publicaciones regulares. En estos años también se empieza advertir un nuevo foco de interés más allá del de la cultura material musical: la relevancia de la dimensión sonora en las culturas de la Prehistoria y de la Antigüedad. El surgimiento de este nuevo campo de investigación estaba estrechamente vinculado al desarrollo de la arqueología de los sentidos, atraída por las experiencias sensoriales de los seres humanos del pasado (ver, por ejemplo, Classen et al., 1994; Cummings, 2002; Tilley, 1994), lo que necesariamente incluía el sentido del oído. Los primeros estudios sobre la importancia cultural de los parámetros acústicos fueron los publicados por légor Reznikoff tanto en solitario (1987, entre otros) como junto a Michel Dauvois (1988), y planteaban una relación directa entre el posicionamiento de algunos motivos de arte parietal paleolítico y puntos especialmente resonantes en el interior de las cuevas. También destacan las propuestas de Steve J. Waller en abrigos y cuevas decoradas europeas y norteamericanas, especialmente las relacionadas con el eco (Waller, 1993a) y los llevados a cabo en monumentos megalíticos británicos (entre otros, Watson, 2001; Watson y Keating, 1999). En los espacios exteriores de la arqueología monumental, salvo unos estudios aislados (Fagg, 1957; Canac, 1967), el interés por el sonido también se despertó a finales de los años noventa (Lubman, 1998; Lubman y Kiser, 2001). Sin embargo, el término arqueoacústica no se popularizó hasta 2003, a partir de la conferencia organizada en ese año por Chris Scarre y Graeme Lawson en el MacDonald Institute for Archaeology y publicada en 2006 (Scarre y Lawson, 2006).

Si bien este no es el lugar para desarrollar ampliamente los diversos métodos de investigación de ambas subdisciplinas, parece ineludible indicar brevemente algunas de las estrategias fundamentales. Por ejemplo, una de las aportaciones más influyentes para

4 Este grupo de estudio fue fundado por las arqueólogas Cajsa Lund, Ellen Hickmann y los conocidos etnomusicólogos John Blacking y Mantle Hood, dato que revela el interés de la etnomusicología por las culturas arqueológicas. 
la metodología de estudio de instrumentos arqueológicos (d'Errico y Lawson, 2006) y que compila otras propuestas anteriores (Lund, 1981; Megaw, 1984) incide en el necesario análisis de su diseño y eficiencia como sistema acústico, así como en la realización de estudios de materiales. Asimismo, defiende la pertinencia de la arqueología musical experimental, que constituye una metodología de interpretación que excede la simple fabricación de objetos técnicamente similares a los arqueológicos, ya que, a partir del estudio minucioso de los materiales, plantea hipótesis en torno a cómo serían los artefactos originales, su cadena operativa, el tiempo invertido y el coste de su manufactura, las técnicas de ejecución y sus posibilidades acústicas y musicales. Si bien el término arqueología experimental fue acuñado en los años 1970 en pleno auge de la Nueva Arqueología o Arqueología Procesual ${ }^{5}$ (Coles, 1973; Reynolds, 1979), este acercamiento al estudio de instrumentos musicales arqueológicos ya disfrutaba un largo recorrido (ver, entre otros, Fétis, 1869: 222-226; Stone, 1878). Finalmente, la mayoría de los autores apoya la búsqueda de paralelos etnográficos y de instrumentos coetáneos, anteriores o posteriores, sin desatender el estudio de fuentes textuales (evidentemente no disponibles en el caso de las culturas musicales paleolíticas, mesolíticas y neolíticas) o iconográficas que verifiquen las hipótesis y propongan contextos performativos (Olsen, 2002; d’Errico y Lawson, 2006; Both, 2009; García Benito y Jiménez Pasalodos, 2011).

Por otro lado, si bien gran parte de los estudios arqueoacústicos mencionados en los párrafos anteriores se basaron en la experiencia sonora de los espacios obtenida de forma natural o medida con pocos medios técnicos (ver, por ejemplo, Reznikoff y Dauvois, 1988; Waller, 1993b; Dauvois y Boutillon, 1994; Reznikoff, 2012; Díaz-Andreu y García Benito, 2012) ${ }^{6}$, en la actualidad se usan métodos de medición derivados de técnicas de la física acústica y en base a los parámetros utilizados para la medición y adecuación acústica de los espacios arquitectónicos (ver, por ejemplo, Fazenda et al., 2017; Díaz-Andreu et al., 2019; Mattioli y Díaz-Andreu, 2017; Mattioli et al., 2017; ver también Díaz-Andreu y Mattioli, 2019). Este método formal de estudio de las características acústicas de los espacios también se complementa con la consideración de los hallazgos arqueológicos e iconográficos, además de con fuentes etnográficas que permiten proponer hipótesis interpretativas (Díaz-Andreu et al., en prensa, 2020) que ponen de relieve la importancia de la dimensión acústica como creadora de conceptos y prácticas culturales.

\section{LAS EVIDENCIAS MÁS ANTIGUAS DE TECNOLOGÍA ACÚSTICA}

Es imposible abordar el cada vez más extenso corpus de hallazgos arqueológicomusicales datados en las primeras etapas de la prehistoria y sus especificidades musicológicas y contextuales en unas pocas líneas, pero algunos ejemplares son testimonios del innegable conocimiento empírico de fenómenos físicos relacionados con la producción de sonido, y su consecuente aplicación en el diseño de tecnología acústica adecuada para controlar la ejecución de diversos tonos que en la actualidad concebimos como musicales. Este apartado pretende ser un breve acercamiento al diseño de los distintos artefactos productores de sonido, en los que se aprovecharon tan eficazmente los diversos principios de funcionamiento físico que en muchas ocasiones han continuado existiendo hasta el presente con pocas variaciones. Además, algunos ejemplos concretos permiten reconocer

5 Algunas de las herramientas que desarrolló son, hoy por hoy, las que predominan en la investigación arqueológica: la etnoarqueología y la arqueología experimental.

6 Algo que por otro lado no era tampoco extraño para los estudiosos del arte rupestre (por ejemplo, los ecos excepcionales remarcados por Arco en el abrigo de arte rupestre de El Civil (1917: 4) o la audibilidad extraordinaria como remarcó Ramón villas en Saltadora (comunicación personal). 
la significativa inversión de tiempo y de recursos dedicados a la musicalidad, así como la transmisión del conocimiento técnico.

Si se clasifican los instrumentos a partir del modo de producción del sonido (Hornbostel y Sachs, [1917] 1961), se puede aceptar la existencia en Eurasia, desde el Paleolítico hasta el Neolítico, de aerófonos, idiófonos y membranófonos y, con reservas, de cordófonos. Los aerófonos incluyen dos tipos: aerófonos de soplo y aerófonos libres. Entre los primeros se distinguen en primer lugar flautas o clarinetes de hueso (ver entre otros, Passemard, 1913; Buisson, 1990 y 1994; Conard, 2007; Conard y Marina, 2008; Conard et al., 2009; Martí Oliver et al., 2001; Zhang et al., 1999; Clodoré-Tissot et al., 2009: 47-53), de dentina de proboscidio (elefante o mamut), que se conoce como marfil (Conard et al., 2004), y de cerámica (Pomberger et al., 2018). También existen silbatos de hueso de ave (entre otros, Ibáñez et al., 2015; Lbova, 2010), de falange de ciervo (entre otros, Chase, 1990 y 2001; Dauvois, 1994: 158-170; Caldwell, 2009; Cârciumaru et al., 2019), de piedra (Beguiristáin y Vélaz, 1998; Luca et al., 2018; Tsuge, 1988) y de cerámica (entre otros, Luca, 2014: figura 13; Turcanu, 2018: 332). Asimismo, se dan hallazgos de trompas de caracola (entre otros, Skeates 1991; Cortese et al., 2004; Villalba et al., 1986; Fritz et al. 2021), y de terracota (entre otros, Coularou et al., 1981; Arnal, 1973: figura 43, n. XXVI). En cuanto a los aerófonos libres, se han identificado una cantidad importante de posibles bramaderas (entre otros, Peyrony, 1930: 22; Barandiarán, 1971 y 2012; Dauvois, 1994: 180; Russell, 2005; ClodoréTissot et al., 2009: 58)7.

El estudio de huellas de uso ha permitido también confirmar la presencia de idiófonos de golpe directo: litófonos fabricados con láminas de sílex auriñacienses (Cross et al., 2002; Blake y Cross, 2008 y 2018), el posible uso idiofónico de formaciones calcáreas en cuevas (entre otros, Dams, 1984 y 1985), e incluso un conjunto de percusión en huesos de mamut decorados proveniente del poblado gravetiense de Mezine, en Ukrania (Bibikov, 1981: 64; Lbova et al., 2013). También hay algunas evidencias incuestionables de idiófonos de golpe indirecto como sonajeros y sonajas. Si bien en el Paleolítico la adscripción de conchas perforadas a tal fin está solamente basada en analogías con instrumentos similares que existen en muchas áreas geográficas (Rubio, 1990: 17; Rubio y Baena, 2002: 167-168), las marcas en la superficie de los conjuntos de dientes perforados del Mesolítico báltico confirman su uso sonoro (Rainio y Mannermaa, 2014). Los sonajeros neolíticos de cerámica (Pavel et al., 2013; Vitezović, 2017: 12; Turcano, 2018: 333) o de caparazón de tortuga (Zhang y Xinghua, 2002: figura 4), apuntan además a la existencia de modelos anteriores de funcionamiento similar, pero fabricados con materiales perecederos. Tampoco existen estudios de huellas de uso de los llamados raspadores (Dauvois, 1994: 182-184; Lund, 1974), aunque su diseño parece adecuado para un uso musical ${ }^{8}$.

En lo que respecta a los membranófonos y los cordófonos, es más difícil afirmar su existencia. La presencia prácticamente universal de tambores en todo el globo hace sospechar de su uso en el Paleolítico (ver también comentarios sobre litófonos más arriba). Algunos investigadores han propuesto que ciertos artefactos de asta, como los bastones perforados paleolíticos en forma de "t" (Kirchner, 1952: 279-282; 131; Larsson, 1988: 147-

7 Aunque algunos son ejemplares ciertamente funcionales, en ocasiones sería necesario revisar las huellas de uso en el orificio en el que se inserta la cuerda. Además, parece que no todos los objetos identificados como bramaderas tienen las proporciones necesarias para funcionar correctamente (Cuartero Monteagudo et al., 2018).

8 Se ha propuesto que el cuerno de bóvido con hendiduras portado por una figura femenina del gravetiense conocida como la "Venus" del abrigo de Laussel (Dordogne, Francia) pudo tratarse de un raspador (Huyge, 1981). 
149; Jiménez Pasalodos y Rainio, 2020), podrían haber sido baquetas de tambores, debido a su similitud con las baquetas del arco circumpolar. Más concluyente parece el empleo musical de ciertos vasos de cerámica sin fondos o con orificios, interpretados como bases de tambores, tanto en Europa, en el área de la Trichterbecher Culture (3400-2800 cal. a.C.) (Wyatt, 2006 y 2010), como en China (Li, 2000: 148; Lawergren, 2006). Tampoco existen pruebas indiscutibles de la existencia de instrumentos de cuerda. El cordófono más sencillo (y probablemente más antiguo) parece derivar del arco, herramienta esencial para la caza, pero las evidencias son débiles. Hay quien apunta que en la cueva con pinturas magdalenienses de Les Trois Frères (Ariège, Francia), uno de los antropomorfos representados con cuerpo y cabeza de bisonte estaría bailando y tocando un arco musical o una flauta de nariz (ver Díaz-Andreu y Mattioli, 2019: 505). Sin embargo, esta lectura ha sido puesta en duda porque es también probable que en realidad la figura esté en cuadrupedia y, por lo tanto, se trate de una persona camuflada intentando cazar (Demouche et al., 1996). Sea como fuere, existen iconografías de instrumentos de cuerda complejos y sofisticados en Mesopotamia y el Levante del Mediterráneo a partir de la segunda mitad del IV milenio a.C., lo que indica una larga tradición constructiva (Duchesne-Guillemin, 1981).

Entre todos los artefactos mencionados se encuentran sin duda algunos ejemplos destacados, fuentes privilegiadas para reflexionar no sólo en torno a la musicalidad en la Prehistoria, sino también al conocimiento acústico y técnico que implica la fabricación de determinados artefactos productores de sonido. Además, a pesar de la distancia geográfica y cronológica de los conjuntos principales y de los cientos de años que separan especímenes en el mismo yacimiento, en ocasiones revelan una sorprendente similitud constructiva e incluso musical (d'Errico y Lawson, 2003: 130). El conjunto de instrumentos más antiguo hallado hasta la fecha y que más repercusión ha tenido en la arqueología general ha sido el de las llamadas "flautas" paleolíticas encontradas en yacimientos europeos (figura 1): una serie de tubos huecos de hueso de ave o de marfil que presentan orificios de digitación. Incuestionables aerófonos de soplo, han sido interpretados mayoritariamente como flautas ${ }^{9}$, a pesar de que el término hace referencia a una forma específica de producción de sonido que implica la necesidad de un bisel, o bien construido en el propio instrumento, o bien generado gracias a una determinada técnica performativa. Sin embargo, no han aparecido tubos con perforaciones que presenten además un bisel construido. De hecho, la evidencia más antigua de flautas con bisel es la del aerófono de Veyreau (figura 2b), datado en el Calcolítico final (Aveyron, Francia), encontrado en una cueva sepulcral junto a restos humanos, y fabricado a partir de cubito de buitre (Fages y Mourer-Chauvire, 1983). Esto significa que, o bien los tubos de hueso perforados paleolíticos podrían haberse tocado como flautas sin bisel (Conard, 2012: 16), de forma oblicua o longitudinal, lo que requiere de una técnica de ejecución que habría conllevado una considerable inversión de tiempo por parte de quien las tocara, o bien a algunos ejemplares se les agregasen lengüetas simples o dobles fabricadas con caña o corteza, lo que les convertiría en aerófonos del tipo clarinete u oboe, con un timbre y un volumen muy distinto al de las flautas (Lawson y d'Errico, 2002: 122; Wyatt, 2012; Mazo et al., 2015) y, en este caso, más sencillos de tocar. Esta última posibilidad supone, por tanto, una muy buena solución técnica al problema de la relativa dificultad de ejecución en flautas sin bisel cuando éstas presentan

9 Aunque existen ejemplares de tubos de hueso que parecen presentar una apertura a modo de bisel, estos nunca tienen orificios de digitación, con lo que habrían sido lo que comúnmente se conoce como silbatos. García Benito considera que, aunque muchos de estos ejemplares sí que serían auténticos silbatos de hueso, en algunos el supuesto bisel no es funcional. Sin embargo, sí que pudieron utilizarse como membranófonos de tipo mirlitón (García Benito, 2013). 
tubos de diámetro pequeño, y las posibilidades de conseguir sonidos a mucho volumen sin demasiado esfuerzo pulmonar. Hay incluso quien sugiere que podrían haberse tocado a modo de trompetas (d'Errico et al., 2003; Wyatt, 2012; Mazo et al., 2015).

Además de esta posible existencia de distintas soluciones para crear la onda estacionaria en el interior del tubo a la que acabamos de referirnos, es importante remarcar que la idea de que un orificio en un tubo acorta la longitud de la columna de aire vibrante y, por tanto, modifica la altura de los sonidos y que, en consecuencia, una serie de orificios permiten producir distintas frecuencias con un solo tubo, implica un conocimiento acústico y tecnológico avanzado (como recuerdan también Lawson y d'Errico, 2002: 121 y d'Errico et al., 2003: 47-48). A menudo se ha propuesto que ciertos tubos de hueso de distintas longitudes, sin perforaciones ni biseles, hubiesen sido utilizados en conjuntos a modo de flautas de Pan, tanto en el Paleolítico (Piette, 1874b), como en el Neolítico (Martí i Oliver, 2001), por su semejanza con instrumentos que existen en varias culturas (figura 2a). Esta solución para producir varios tonos es sin duda más evidente, ya que la relación entre la longitud del tubo y la altura de los sonidos es fácilmente observable. Sin embargo, algunos intervalos, ornamentos o determinados efectos acústicos son prácticamente imposibles de ejecutar, especialmente a cierta velocidad. Sin embargo, una flauta fabricada con un solo tubo, pero con varios orificios de digitación, ofrece diferentes posibilidades musicales. No obstante, además de idear un sistema que acorte el tubo al antojo del intérprete, el cual puede controlar fácilmente esa variación de longitud clausurando agujeros con los dedos de ambas manos, llegar a un diseño adecuado no es tan sencillo. Por ejemplo, si la perforación que se realiza tiene el mismo diámetro del tubo, aunque el sonido producido equivale al de un tubo abierto a esa distancia, es muy difícil de cerrar. Por otro lado, los agujeros demasiado pequeños no afectan a la longitud de la columna de aire. Las perforaciones de tamaño medio, sin embargo, son cómodas de taponar y conllevan reducciones parciales de la longitud de la columna de aire, lo que las hace funcionalmente adecuadas. En consecuencia, dada la complejidad de las flautas paleolíticas encontradas, estas no pueden sino reflejar una tradición de instrumentos anteriores probablemente fabricados con materiales blandos. A esto apunta también la explicación de la fabricación por rebajado de los orificios en ejemplares con cronologías muy diversas, en lugar de por perforación (Mazo et. al., 2015). Aunque hay quien defiende que esos rebajes facilitarían la adhesión neumática de los dedos de los intérpretes, especialmente en manos muy castigadas (Lawson y d'Errico, 2002: 123), es también posible que esta técnica de fabricación esté derivada de la construcción de aerófonos en otros materiales para los que la perforación no es adecuada (Mazo et al., 2015).

A partir del Neolítico se siguen construyendo aerófonos de hueso con múltiples orificios de digitación (Clodoré-Tissot et al., 2009: 47-53), pero en algunas ocasiones hay indicios de un desarrollo constructivo que buscaba aumentar las posibilidades musicales. Este es el caso de las flautas provenientes de las tumbas neolíticas del yacimiento de Jiahu (figura 2c), en la provincia china de Henan (7.000-5.800 a. C.), a veces acompañadas de sonajeros de caparazón de tortuga (Zhang et al., 1999; Zhang y Jinghua, 2002; Zhang et al., 2004). En total, se han recuperado unos treinta ejemplares fabricados en hueso de grulla de coronilla roja, con entre tres y ocho orificios de digitación. Su distribución cronológica permite suponer modificaciones en la organización de los orificios para proporcionar mayor número de intervalos, así como una tendencia a la estandarización de las afinaciones y las escalas, probablemente para que varios instrumentos tocasen a la vez (Zhang et al., 2004). Pero la investigación y experimentación acústica no solo se refleja en estos artefactos. Durante el Neolítico, en toda Eurasia se documentan instrumentos musicales de cerámica, los primeros de una larga práctica que continúa en muchas culturas tradicionales del 
continente, tanto de aerófonos (Luca, 2014: figura 13; Turcanu, 2018: 332; Pomberger et al., 2018), como de otras categorías organológicas que podrían ser un reflejo de instrumentos similares anteriores fabricados con materiales orgánicos, como sonajeros (Pavel et al., 2013; Vitezović, 2017: 12; Turcano, 2018: 333) y trompas de cerámica, probablemente inspiradas en trompas de cuerno natural (Coularou et al., 1981; Arnal, 1973: figura 43, n. XXVI).

\section{LA MÚSICA COMO CULTURA EN LA PREHISTORIA: UNA MIRADA DESDE LA MATERIALIDAD}

Los casos anteriores son un ejemplo del significativo conocimiento acústico y tecnológico que necesariamente tuvieron las culturas paleolíticas y neolíticas que fabricaron artefactos sonoros. Pero, además, tanto los instrumentos como los contextos arqueológicos permiten reflexionar en torno a los comportamientos simbólicos y las prácticas culturales asociadas con la música.

De nuevo, los aerófonos de hueso son una fuente privilegiada. Si su diseño acústico es sin duda complejo, una vez entendido el principio de funcionamiento su manufactura sobre huesos de ave no presenta muchos problemas (Mazo et al., 2015: 86), ya que son naturalmente huecos y requieren poca intervención para convertirse en aerófonos de soplo. Sin embargo, en las cuevas paleolíticas del Jura de Suabia (Alemania) también han aparecido aerófonos construidos con marfil. Acústicamente, los materiales de construcción de un aerófono no influyen en el sonido resultante, $y$, sin embargo, la manufactura en marfil es mucho más dificultosa. De hecho, estos aerófonos, todos datados en el Auriñaciense, son acaso los objetos más laboriosos de fabricar de todos los conservados con cronología paleolítica. El ejemplar mejor preservado, que además resulta ser el instrumento musical más antiguo documentado (Higham et al., 2012) ${ }^{10}$, proveniente de la cueva Geißenklösterle (de en torno a $42.000 \mathrm{cal} \mathrm{AP)} \mathrm{(figura} \mathrm{1b).} \mathrm{El} \mathrm{fragmento} \mathrm{revela} \mathrm{cómo,} \mathrm{utilizando} \mathrm{exclusivamente}$ industria lítica, se hubo de tallar un fino cilindro de marfil de mamut, que posteriormente se cortó longitudinalmente y se vació. Tras abrir los orificios de digitación, se realizaron muescas en ambas mitades para facilitar la unión de las piezas con algún tipo de resina (Conard et al., 2004: 455-457). Si bien solo se aprecian tres orificios, parece probable que el instrumento contara con alguno más. Además, existe evidencia de continuidad tecnológica y simbólica en otros tres aerófonos de marfil encontrados en las cuevas vecinas de Hohle Fels y de Vogelherd, y datados al menos en un par de milenios después (Münzel et al., 2002; Conard, 2007; Conard y Malina, 2008; Conard et al., 2009), lo que revela la persistencia de la tradición. ¿Por qué se fabricaron estos difíciles tubos de marfil si la construcción de instrumentos con hueso de buitre era relativamente rápida y muy eficaz? Lo más probable es que sean determinados conceptos simbólicos los que justifiquen esta considerable inversión de tiempo y tecnología.

Por otro lado, aunque la mayoría de los aerófonos con varios orificios de digitación recuperados en distintas cronologías desde el Auriñaciense están fabricados con huesos de rapaces o de cisne, una serie de marcas o decoraciones en la superficie también han permitido proponer hipótesis sobre las prácticas culturales asociadas. En Isturitz (PyrénéesAtlantiques), se han identificado en torno a una veintena de fragmentos realizados en huesos de buitre y rapaces diurnas, con entre 1 y 4 orificios de digitación (Buisson, 1990 y 1994). Los ejemplares cubren una cronología desde el Auriñaciense hasta el Magdaleniense, aunque la gran mayoría se localizan en estratos gravetienses (por ejemplo, figuras 1e y 1d).

10 El hueso de oso perforado de Divje Babe interpretado como una posible flauta neandertal datada en el Musteriense (Turk, 1997; Turk et al., 2020) ha sido puesto en duda en múltiples ocasiones ya que parece que las perforaciones fueron realizadas por un carnívoro (d’Errico et al., 1998a y b; Cajus, 2015). 
En las cuevas alemanas del Jura de Suabia son también varios los ejemplares en hueso de ave auriñacienses: en Hohle Fels se halló un ejemplar de radio de buitre con al menos cinco orificios (figura 1c), que apareció junto a una figura femenina de marfil (Conard et al., 2009), lo que ha permitido sugerir que ambos elementos tuvieron un uso conjunto, quizá ritual (Floss, 2015); en la de Vogelherd aparecieron dos fragmentos de flautas de hueso de ave (Conard, 2007), y en Geisserklosterle, se encontraron dos flautas fabricadas con hueso de cisne (Münzel et al., 2002; Conard et al., 2004) (figura 1a). Tanto los ejemplares de los conjuntos de Isturitz como los del Jura de Suabia presentan varias líneas incisas que no tienen ninguna explicación tecnológica, salvo en el caso de una serie de muescas en los ejemplares de marfil para facilitar el pegado de sus dos secciones. Lawson y d'Errico han propuesto que, ya que las líneas paralelas incisas longitudinalmente en algunos ejemplares de Isturitz fueron realizadas con distintas herramientas, movimientos, espaciados, técnicas y orientaciones, lo más probable es que se hicieran en distintos momentos. Por tanto, proponen que, más que decoraciones o mensajes con información codificada, la realización misma de los grabados pudo formar parte de la performance musical o ritual asociada con el instrumento (2002: 128-129), e incluso aventuran que la incidencia de marcas en lugares cercanos a los orificios de digitación pudo suponer algún tipo limitado de notación musical (2002: 129-130). De hecho, ciertas incisiones en la superficie parecen estar relacionadas con la distribución de los orificios en algunos ejemplares tanto de Isturitz como de las cuevas del Jura de Suabia (Lawson y d'Errico, 2002: 124).

Los contextos arqueológicos también pueden servir para valorar los contextos performativos. Por ejemplo, con la excepción de un ejemplar, los aerófonos de Isturitz provienen de la llamada Grande Salle (Buisson, 1990 y 1994), un espacio con una acústica remarcable, como se ha visto en otras cuevas paleolíticas francesas (Reznikoff, 2008). Es sugestivo pensar que durante todo el Gravetiense y quizá en algún momento en el Solutrense y Magdaleniense, grupos o individuos fueran a la cueva atraídos por la reverberación de ese espacio, y su adecuación para la práctica musical. De hecho, parece que la cueva de Isturitz fue un lugar de reunión temporal de comunidades del norte de la península ibérica y de los Pirineos occidentales, que se congregaban en determinados momentos del año (Normand et al., 2012: 178-179). Probablemente, la práctica musical tuvo un importante papel en la socialización y el establecimiento de relaciones entre los grupos, quizás relacionada con otros comportamientos de posible significado ritual (ver, por ejemplo, Garate et al., 2019).

En el caso de los aerófonos neolíticos de Jiahu (Zhang et al., 1999; Zhang y Xinghua, 2002; Xinghua, 2002; Zhang et al., 2004), el contexto funerario de los hallazgos también ejemplifica la importancia social de la música, en este caso por su relación con el posible estatus y especialización de los individuos que fueron enterrados con las flautas y los sonajeros de caparazón de tortuga, además de otros elementos marcadores de estatus (Zhang y Cui, 2013: 2002-204). Sus descubridores apuntan a la importancia del material elegido para su construcción, tanto al hueso de grulla, con significados cosmológicos en China (Zhang et al., 2004), y asociados con la flauta en mitos registrados a partir del primer milenio d. C., como a las tortugas, ya que hay evidencias de su sacrificio ritual, lo que respalda significados simbólicos específicos. Su utilización para fabricar sonajeros quizá pueda incluso ser precursora de algunos ritos de adivinación utilizados en épocas posteriores (Zhang y Cui, 2013: 207-208). La diferencia de estatus de los enterramientos de Jiahu no parece coincidir con una estratificación social marcada, sino más bien con la especialización de ciertos individuos. En la primera fase, unas pocas tumbas concentran un alto número de artefactos sonoros, como es el caso de la tumba M344, un enterramiento masculino sin cráneo, acompañado de ocho sonajeros de caparazón de tortuga y dos flautas. Los investigadores apuntan a que pudiera tratarse de un especialista ritual o chamán (Zhang y Cui, 2013: 208). 
Figura 1. Ejemplos de aerófonos de hueso paleolíticos con orificios de digitación a. Aerófono de radio de cisne de Geißenklösterle
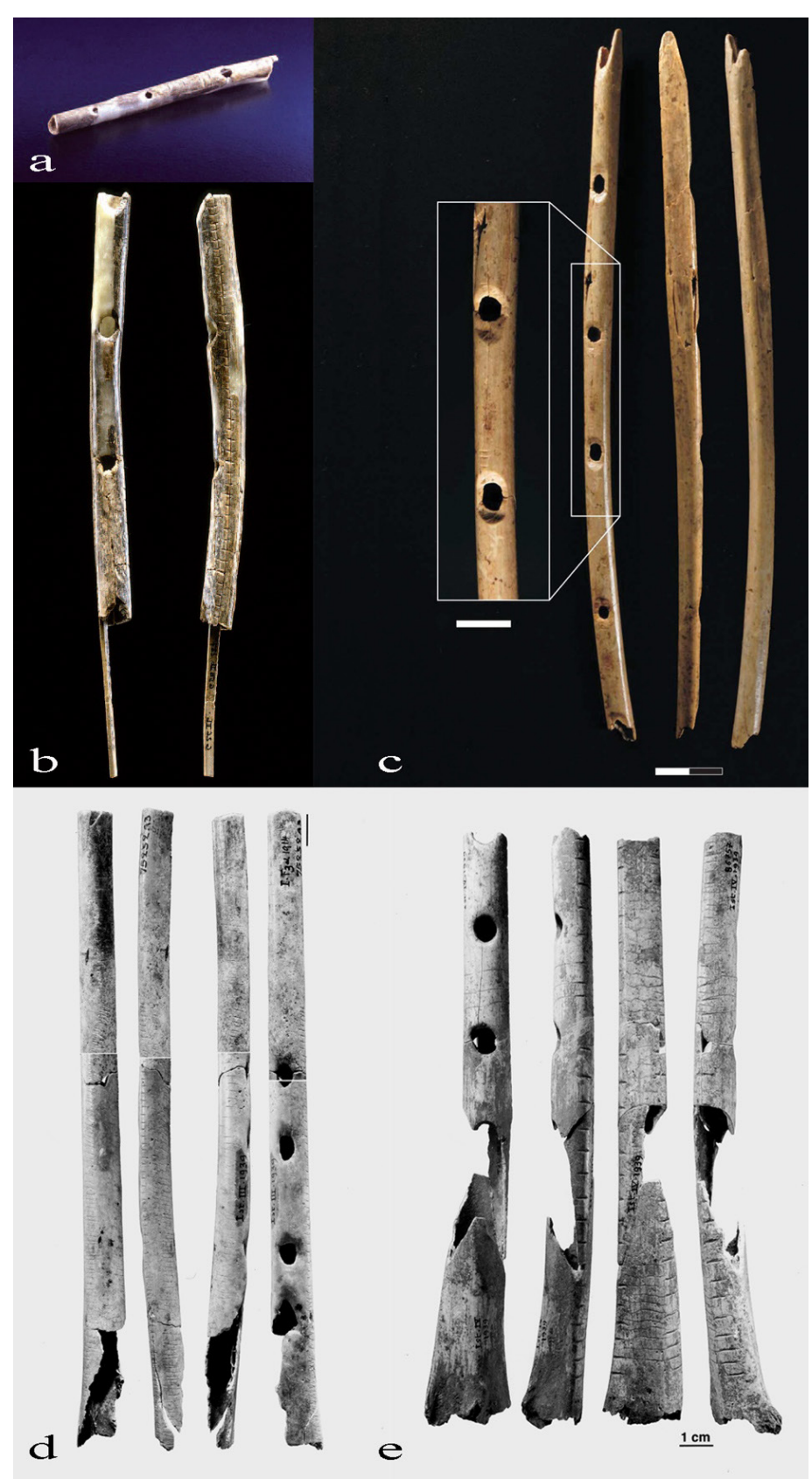

Fuente: H. Jensen, (c) Universidad de Tubinga (Conard, 2012: 15). b. Aerófono de marfil de Geißenklösterle. Foto: J. Lipták, ( ) Universidad de Tubinga (Conard, 2012: 15). c. Aerófono de radio de buitre de Hohle Fels (Conard et al., 2009: fig. 1) d. Dos aerófonos de ulna de buitre de Isturiz unidos por Buisson: tubo superior gravetiense $n^{\circ} 75252-A 3$ y tubo inferior auriñaciense final $n^{\circ} 83888$ (a). Musée des Antiquités Nationales, Saint Germain-en-Laye. Foto: F. d'Errico (Lawson y d'Errico, 2002: lám. II). e. Aerófono de ulna de buitre de Isturitz. Tubo no 86757 (a) unido por Buisson al tubo no DB 5.1. Musée des Antiquités Nationales, Saint Germain-en-Laye. Foto: F. d'Errico (Lawson y d'Errico, 2002: lám. III). 
Figura 2. Ejemplos de flautas neolíticas y calcolíticas.
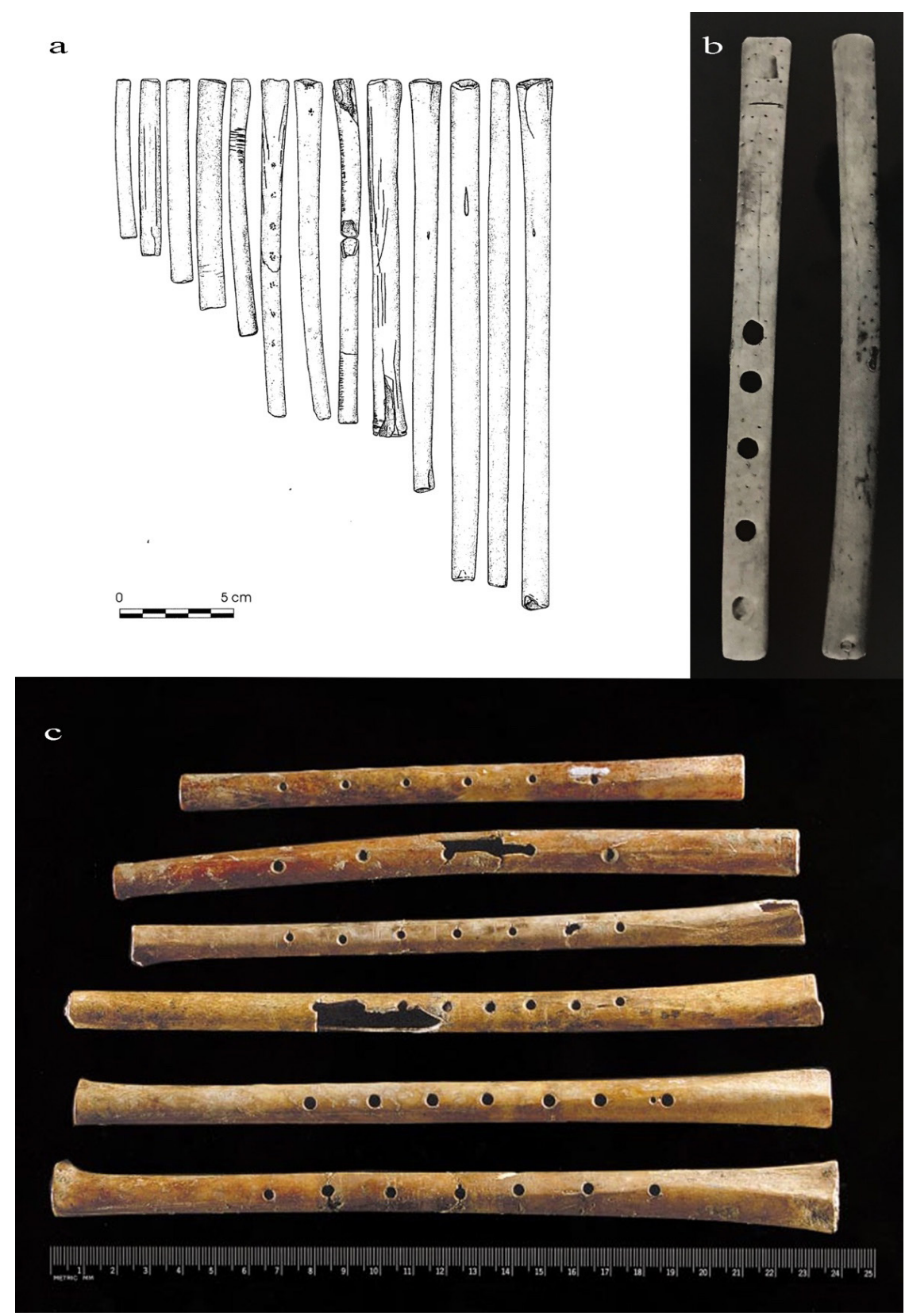

Fuente: Zhang et al., 1999: fig. 1).a. Tubos de hueso enteros de buitre de la Cova de l'Or reconstruidos como una flauta de Pan (Martí Oliver et al, 2001: fig. 8). b. Flauta calcolítica de Veyreau (Fages y MourerChauviré, 1983: fig. 3). c. Algunas flautas neolíticas de Jiahu (de arriba abajo) M341:2, M341:1, M78:1, M253:4, M282:20, M282:21 
Estos ejemplos son una buena muestra de que las tradiciones musicales de las primeras etapas de la Prehistoria euroasiática mostraban muchos de los conceptos y los comportamientos culturales en torno a la música que existen en numerosas prácticas tanto históricas como contemporáneas, y desvelan que probablemente fueron tan ricas, variadas y complejas como las tradiciones vivas que ha estudiado la etnomusicología y la antropología (ver, por ejemplo, Merriam, 1964; Blacking, 1973; Seeger, 1987; Feld, 1982; Nettl, 2005; Levin y Süzükei, 2010; Lewis, 2013; Trehub et al., 2015). Canciones, melodías y danzas seguramente acompañaron a las personas desde antes de su nacimiento, primero con las músicas que escuchaban en el vientre materno y, después, con las nanas que cantaban los hombres y mujeres encargados de cuidar a los recién nacidos. Además, las canciones infantiles enseñarían a las niñas y a los niños su lugar en el mundo y les permitían fortalecer sus vínculos sociales, y también beneficiarían su desarrollo cognitivo y emocional. A la vez que aprendían su lengua natal y memorizaban el conocimiento cultural, los mitos y las historias, se impregnarían de la estética musical de sus comunidades. A lo largo de su vida, la música y la danza se utilizarían en diversos rituales en los que se fomentaría la cohesión grupal e intergrupal, se mostraría la solidaridad, la empatía y la reciprocidad, a la vez que se reforzaría la identidad cultural compartida. Esas ocasiones serían también propicias para asegurar la reproducción sexual y social. La práctica musical estimularía entonces, como hace ahora, los sentimientos de placer gracias a la segregación de dopamina (Salimpoor et al., 2011), favoreciendo una resolución pacífica y efectiva de las tensiones sociales y de los retos emocionales y angustias trascendentales a los que nos enfrentamos los seres humanos. Igualmente, acaso en estos momentos de liminalidad algunos bailes y canciones sirviesen para retar ritualmente normas sociales, permitiendo el desahogo emocional y la aceptación social de formas diversas de ser persona. En algunas ocasiones, la música sería también canalizadora de lo sagrado, quizás de la mano de especialistas rituales, facilitando estados de trance o estimulando el sentimiento de trascendencia y de comunicación con aquello que se escapaba de la realidad cotidiana y que permitía explicar el mundo de manera satisfactoria, así como influir en él cuando era necesario, quizás para agradar a los espíritus y propiciar una buena caza, o para evitar fenómenos meteorológicos adversos. O tal vez para alejarlos y así impedir o curar enfermedades. Muchas veces constituiría sin embargo un mero entretenimiento estético, que ayudaba a pasar las horas tediosas o propiciaba la coordinación en arduas tareas colectivas.

\section{CONCLUSIONES}

Si bien los restos materiales de la musicalidad de los primeros grupos humanos en Eurasia son solo un pálido reflejo de unas prácticas que incluirían cantos, danzas, palmas, instrumentos fabricados con materiales orgánicos, representaciones de historias, indumentarias, adornos, y otras estimulaciones sensoriales, son suficientes para afirmar que las culturas paleolíticas y neolíticas euroasiáticas mostraron rasgos propios de tradiciones musicales complejas y desarrolladas. El estudio tecnológico de los artefactos evidencia el avanzado conocimiento empírico de distintos fenómenos físicos relacionados con el sonido, así como una tradición técnica que permitió la producción de instrumentos musicales muy efectivos, hasta el punto de que las principales categorías organológicas actuales estaban ya representadas. Además, hay ciertos indicios que apuntan a una remarcable continuación tecnológica y musical, que perduró durante varios milenios, y que abarcó áreas geográficas alejadas, lo que lleva a reflexionar en torno a la significativa conservación cultural de prácticas asociadas con la música. La considerable inversión de tiempo y recursos también permite aventurar la importancia de la música en las sociedades prehistóricas, y, en muchas ocasiones, puede revelar una relación entre música y significados simbólicos o prácticas 
rituales. Finalmente, los contextos arqueológicos parecen confirmar estas interpretaciones, y permiten hipotetizar algunos de los usos y funciones de la música en aquellas comunidades, entre los que probablemente se encontraran diversos tipos de rituales relacionados con distintas concepciones cosmológicas o con prácticas que favorecieron la cohesión social.

\section{BIBLIOGRAFÍA}

Arbaud, P. D. (1857), Recherches sur la flûte ancienne, Paris, Garnier frères.

Arco, L. D. (1917), "Descubrimiento de pinturas rupestres del Barranco de la Valltorta", Boletín de la Real Academia de la Historia, 71, pp. 5-17.

Arnal, J. (1973), “I. Etude archéologique. Le Lebous à Saint-Mathieu-de-Tréviers (Hérault). Ensemble du Chalcolithique au Gallo-Romain", Gallia Préhistoire, 16, 1, pp. 131-193.

Ball, R. (1844), "On the Crotal", Proceedings of the Royal Irish Academy (1836-1869), 3, pp. 135-136.

Barandiarán, I. (1971), “'Bramaderas' en el Paleolítico Superior Peninsular”, Pyrenae, 7, pp. 7-18.

- (2012), "Forma vs. Función en tipología prehistórico: el caso de las bramaderas óseas magdalenienses", Veleia, 29, pp. 307-334.

B.B. [posiblemente Benjamin Bartlet] (1763), Texto explicativo de la Lámina XX, Vetusta Monumenta II, London, Society of Antiquaries.

Bégouén, C. H. y Breuil, A. H. (1958), Les cavernes du Volp: Trois-Frêreres, Tud d'Audoubert, Paris, Arts et métiers graphiques.

Beguiristáin, M. A. y Vélaz, D. (1998), "Objetos de adorno personal en el dolmen de Aizibita (Cirauqui, Navarra)", Cuadernos de Arqueología de Navarra, 6, pp. 7-31.

Bibikov, S. N. (1981), Drevneishii Muzykalnyi Kompleks iz Kostei Mamonta: Ocherk Materialnoi i Dukhovnoi Kultury Paleoliticheskogo Cheloveka, Kiev, Naukova Dumka.

Blacking, J. (1974), How musical is man?, Seattle, London, University of Washington press.

Blake, E. y Cross, I. (2008), "Flint Tools as Portable Sound-Producing Objects in the Upper Palaeolithic Context: An Experimental Study", P. Cunningham, J. Heeb y R. Paardekooper (eds.), Experiencing Archaeology by Experiment: Proceedings of the Experimental Archaeology Conference, Exeter 2007, Oxford, Oxbow Books, pp. 1-19.

- (2015), "The Acoustic and Auditory Contexts of Human Behavior", Current anthropology, 56, 1, pp. 81-103.

Both, A. A. (2009), "Music archaeology: some methodological and theoretical considerations", Yearbook for Traditional Music, 41, pp. 1-11.

Buisson, D. (1990), "Les flûtes paléolithiques d'Isturitz (Pyrénées-Atlantiques)", Bulletin de la Société préhistorique française, 87, 10-12, pp. 420-432.

(1994), "Les flutes paleolitiques d'Isturitz", A. Bellis y C. Homo-Lechner (eds.), La pluridisplinarité en archéologie musicale. (IVs rencontres internationales d'archéologie musicale de l'ICTM), Paris, Maison des Sciences de l'Homme, pp. 259-275.

Caldwell, D. (2009), "Palaeolithic Whistles or Figurines? A Preliminary Survey of Pre-historic Phalangeal Figurines", Rock Art Research, 26, 1, pp. 65-82.

Canac, F. (1967), L'acoustique des théâtres antiques, Paris, CNRS.

Carmen, P., Cosmin, S., Florin, C. y Roxana, B. (2013), "X-ray computed tomography investigations of Cucuteni ceramic statuettes", Documenta Praehistorica, 40, pp. 323332.

Chase, P. G. (1990), "Sifflets du Paléolithique moyen (?). Les implication d'un coprolithe actuel", Bulletin de la Société Préhistorique Française, 87, 6, pp. 165-167.

(2001), "Punctured reindeer phalanges from the Mousterian of Combe Grenal", Arheološky vestnik, 52, pp. 17-23. 
Classen, C., Howes, D. y Synott, A. (1994), Aroma: The Cultural History of Smell, London, Roudledge.

Clodoré-Tissot, T., Le Gonidec, M.-B., Ramseyer, D. y Anderes, C. (2009), Instruments sonorres du Néolithique à l'aube de l'Antiquité, Paris, Éditions Societé préhistorique française.

Coles, J. (1973), Archaeology by experiment London, Hutchinson.

Conard, N. J. (2007), "Les flûtes aurignaciennes des grottes Geißenklösterle et du Vogelherd (Jura souabe)", H. Floss y N. Rouquerol (eds.), Les chemines de l'art aurignacien en Europe / Das Aurignacien und die Anfänge der Kunst in Europa, Cambridge, Cambridge University Press, pp. 353-362.

- (2012), "La importancia evolutiva de las primeras evidencias de arte figurativo y los instrumentos musicales de las cuevas del suroeste de Alemania", I. Escobar y B. Rodríguez Álvarez (eds.), Arte sin artistas. Una mirada al Paleolítico: [exposición celebrada en el] Museo Arqueológico Regional, Alcalá de Henares, Madrid, de diciembre de 2012 a abril de 2013, Madrid, Museo Arqueológico Regional, pp. 439462.

Conard, N. J. y Malina, M. (2008), "New Evidence for the Origins of Music from the Caves of the Swabian Jura", A. A. Both, R. Eichmann, E. Hickmann y L.-C. Koch (eds.), Studien zur Musikarchäologie VI, Rahden, Marie Leidorf, pp. 13-22.

Conard, N. J., Malina, M. y Münzel, S. C. (2009), "New Flutes Document the Earliest Musical Tradition in Southwestern Germany", Nature, 460, pp. 737-740.

Conard, N. J., Malina, M., Münzel, S. C. y Seeberger, F. (2004): "Eine Mammutelfenbeinflöte aus dem Aurignacien des Geißenklösterle: Neue Belege für eine musikalische Tradtion im Frühen Jungpaläolithikum auf der Schwäbischen Alb", Archäologisches korrespondenzblatt, 34, pp. 447-462.

Cortese, G. E., Del Lucchese, A. y Garibaldi, P. (2004), "Charonia sp., uno strumento musicale del Neolitico?", Preistoria Alpina, 40, pp. 91-96.

Coularou, J., Vaton, J. y Vincent, A. (1981), "Une trompe en céramique dans un niveau chalcolithique (Abri $n^{\circ} 7$ de Brugas, Vallabrix, Gard), Bulletin. Société Préhistorique. Française, 78, 4, pp. 106-107.

Cross, I., Zubrow, E. y Cowan, F. (2002), "Musical behaviours and the archaeological record: a preliminary study", J. R. Mathieu (ed.), Experimental Archaeology: replicating past objects, behaviors and processes, Oxford, British Archaeological Reports, pp. 25-34.

Cuartero Monteagudo, F., Terradillos Bernal, M., Canales Camarero, D., Alonso Alcalde, R. y Alcaraz-Castaño, M. (2018), “Una aproximación a la problemática de los zumbadores magdalenienses a partir de la experimentación: influencia de las proporciones métricas de la sonoridad", Bulletí Arqueològic, 5, 40, pp. 219-224.

Cummings, V. (2002), "Experiencing texture and transformation in the British Neolithic", Oxford Journal of Archaeology, 21, 3, pp. 249-261.

d'Errico, F., Henshilwood, C., Lawson, G., Vanhaeren, M., Tillier, A.-M., Soressi, M., Bresson, F., Maureille, B., Nowell, A., Lakarra, J., Backwell, L. y Julien, M. (2003), "Archaeological Evidence for the Emergence of Language, Symbolism, and Music-An Alternative Multidisciplinary Perspective", Journal of World Prehistory, 17, 1, pp. 1-70. d'Errico, F. y Lawson, G. (2006), "The Sound Paradox. How to assess the acoustic significance of archaeological evidence?", C. Scarre y G. Lawson (eds.), Archaeoacustics, Cambridge, Cambridge University Press, pp. 41-57.

d'Errico, F., Villa, P., Pinto Llona, A. y Ruiz Idarraga, R. R. (1998a), "A Middle Palaeolithic origin of music? using cave-bear bone accumulations to assess the Divje Babe I bone 'flute"', Arts y Humanities, 72, pp. 65-79. 
- (1998b), "La 'flûte' de Divje Babe et les accumulations naturelles d'ossements d'ours des cavernes", J.-P. Brugal, L. Meignen y M. Patou-Mathis (eds.), Économie préhistorique: les comportements de subsistenace au Paléolithique. Actes de SVIII rencontres internationales d'Archéologie et D'Histoire d'Antibes, Antibes, Éditions APDCA, Centres de recherches archéologiques Ville d'Antibes, pp. 85-104.

Dams, L. (1984), "Preliminary findings at the 'Organ' Sanctuary in the Cave of Nerja, Malaga, Spain", Oxford Journal of Archaeology, 3, 1, pp. 1-14.

- (1985), "Palaeolithic lithophones: descriptions and comparisons", Oxford Journal of Archaeology, 4, 1, pp. 31-46.

Daussoigne-Mehul, J. L. (1848), "L’archéologie musicale", Revue et gazette musicale de Paris.

Dauvois, M. (1994), "Son et musique paléolithique", A. Bellis y C. Homo-Lechner (eds.), La pluridisplinarité en archéologie musicale. (IVs rencontres internationales d'archéologie musicale de l'ICTM), Paris, Maison des Sciences de l'Homme, pp. 151-206.

Dauvois, M. y Boutillon, X. (1994), "Caractérisation acoustique des grottes ornées Paléolithiques et de leurs lithophones naturels", A. Bellis y C. Homo-Lechner (eds.), La pluridisplinarité en archéologie musicale. (IVs rencontres internationales d'archéologie musicale de l'ICTM), Paris, Maison des Sciences de l'Homme, pp. 151-206.

Demouche, F., Slimak, L. y Deflandre, D. (1996), "Nouvelle approche de la gravure du petit sorcier à l'arc musical de la grotte des Trois Frêres: Ariège", Préhistoire Anthropologie Méditerranéennes, 5, pp. 35-37.

Díaz-Andreu, M. y García Benito, C. (2012), "Acoustics and Levantine Rock Art: Auditory Perceptions in La Valltorta Gorge (Spain)", Journal of Archaeological Science, 39, pp. 3591-3599.

Díaz-Andreu, M., García Benito, C., Lazarich, M., Ramos, A., Cruz, M. J. y Gao, Q. (2013), "Archaeoacoustics and the Location of Rock Art in Spain", P. Whitehead (ed.), IFRAO 2013 Proceedings, Glendale, Arizona, American Rock Art Research Association, pp. 661-667.

Díaz-Andreu, M., Farina, A., Armelloni, E., Coltofean, L., Picas, M. y Mattioli, T. (2019), "Acoustic effects at prehistoric landscapes: an archaeoacoustics analysis of rock art sites from Western Mediterranean", M. Vorländer y J. Fels (eds.), Proceedings of the 23rd International Congress on Acoustics 9 to 13 September 2019 in Aachen, Germany, Berlin, German Acoustical Society, pp. 281-287. (en línea)

Díaz-Andreu, M. y Mattioli, T. (2019), "Rock Art, music and acoustics: a global overview", B. David y I. J. McNiven (eds.), The Oxford Handbook of the Archaeology and Anthropology of Rock Art, Oxford, Oxford University Press, pp. 503-528.

Díaz-Andreu, M., Mattioli, T. y Rainsbury, M. (2021), "The cultural understanding of sound in rock art landscapes: the limits of interpretation", J. M. Gjerde y M. S. Arntzen (eds.), Perspectives on Differences in Rock Art, Proceedings of the ACRA III - The Alta Conference on Rock Art III, Alta, Norway 15-18 September 2015. London, Equinox, pp. 244-265.

Diedrich, C. G. (2015), "Neanderthal bone flutes: simply products of Ice Age spotted hyena scavenging activities on cave bear cubs in European cave bear dens", Royal Society Open Science, 2, 4, pp. 140022.

Duchesne-Guillemin, M. (1981), "Music in Ancient Mesopotamia and Egypt", World Archaeology, 12, 3, pp. 287-297.

Fages, G. y Mourer-Chauvire, C. (1983), "La flute en os d'oiseau de la grotte sepulcrale de Veyreau (Aveyron) et inventaire des flútes préhistoriques d'Europe", F. Poplin (ed.), La Faune et L'Homme préhistorique: dix études en hommage á Jean Bouchoud, Paris, 
Société Préhistorique Française, pp. 95-103.

Fagg, B. (1957), "Rock gongs and rock slides", Man, 57, pp. 30-32.

Fazenda, B., Scarre, C., Till, R., Jiménez Pasalodos, R., Rojo Guerra, M., Tejedor, C., Peredo, R. O., Watson, A., Wyatt, S., García Benito, C., Drinkall, H. y Foulds, F. (2017), "Cave acoustics in prehistory: Exploring the association of Palaeolithic visual motifs and acoustic response", Journal of the Acoustical Society of America, 142, 3, pp. 1332.

Feld, S. (1982), Sound and Sentiment: Birds, Weeping, Poetics, and Song in Kaluli expression, University of Pennsylvania Press.

Fétis, F.-J. (1869), Histoire générale de la musique depuis les temps les plus anciens jusqu'à nos jours, Paris, Firmin-Didot.

Floss, H. (2015), "The Oldest Portable Art: the Aurignacian Ivory Figurines from the Swabian Jura (Southwest Germany)", Palethnologie, 7, http://journals.openedition. org/palethnologie/888

Fritz, C., Tosello, G., Fleury, G., Kasarhérou, E., Walter, P., Duranthon, F., Gaillard, P. y Tardieu, J. (2021), "First record of the sound produced by the oldest Upper Paleolithic seashell horn", Science Advances, 7, 7, pp. eabe9510.

Garate, D., Labarge, A., Rivero, O., Intxaurbe, I., Barshay-Szmidt, C. y Normand, C. (2019), "Another bone in the wall: towards a characterisation of the objects placed in wall fissures at Isturitz cave (Pyrénées-Atlantiques, France)", Archaeological and Anthropological Sciences, 11, 12, pp. 6875-6887.

García Benito, C. (2013), "The experimentation process in the skin of the researcher: the case of the Isturitz, Le Placard and Saint-Marcel 'Whistles'", G. Compañy, J. Fonte, B. Gómez-Arribas, L. Moragón y J. M. Señorán (eds.), Actas de las $V$ Jornadas de Jóvenes en Investigación Arqueológica, Arqueología para el siglo XXI, Santiago de Compostela, mayo de 2012, Santiago de Compostela, JAS Arqueología, pp. 187-191.

García Benito, C. y Jiménez Pasalodos, R. (2011), "La música enterrada: Historiografía y Metodología de la Arqueología Musical", Cuadernos de Etnomusicología de la SIBE, 1, pp. 80-108.

Gourlay, K. A. (1984), "The Non-Universality of Music and the Universality of Non-Music", The World of Music, 26, 2, pp. 25-39.

Higham, T., Basell, L., Jacobi, R., Wood, R., Ramsey, C.B. y Conard, N.J. (2012), “Testing models for the beginnings of the Aurignacian and the advent of figurative art and music: the radiocarbon chronology of Geißenklösterle", Journal of Human Evolution, 62, pp. 664-676.

Huyge, D. (1991), "The 'Venus' of Laussel in the light of Ethnomusicology", Archeologie in Vlaanderen, 1, pp. 11-18.

Ibáñez, J. J., Salius, J., Clemente-Conte, I. y Soler, N. (2015), "Use and Sonority of a 23,000-Year-Old Bone Aerophone from Davant Pau Cave (NE of the Iberian Peninsula)", Current Anthropology, 56, 2, pp. 282-289.

Jiménez Pasalodos, R. (2020): Las trompetas de cerámicas celtibéricas: una aproximación al patrimonio arqueológico musical de la Segunda Edad del hierro. Tesis Doctoral, Universidad de Valladolid.

Jiménez Pasalodos, R. y Rainio, R. (2020), "Missing Membranophones? Traditional Drumbeaters in Northern Eurasia and Possible Prehistoric Parallels in European Archaeological Collections", G. Kolltveit y R. Rainio (eds.), The Archaeology of Sound, Acoustics and Music, Berlin, Ekho Verlag, pp. 233-276.

Kirchner, H. (1952), "Ein Archäologischer Beitrag Zur Urgeschichte Des Schamanismus", Anthropos, 47, 1, pp. 244-286.

Larsson, L. (1988), Ett fångstsamhälle för 7000 sedan: Boplatser och gravar i Skateholm, 
Lund, Signum.

Lawergren, B. (2006), "Neolithic Drums in China”, E. Hickmann y R. Eichmann (eds.),Studien zur Musikarchaologie V, Radhen, Marie Leidorf, pp. 109-127.

Lawson, G. y d'Errico, F. (2002), "Microscopic, Experimental and Theoretical Re-Assessment of Upper Palaeolithic Bird-Bone Pipes from Isturitz, France: Ergonomics of Design, Systems of Notation and the Origins of Musical Traditions", E. Hickmann, A. Kilmer y R. Eichmann (eds.), Studien zur Musikarchäologie III, Radhen, M. Leidorf, pp. 119-142.

Lbova, L. (2010), "Evidence of modern human behavior in the Bailkal zone during the early upper paleolithic period", Bulletin of the Indo-Pacific Prehistory Association, 30, pp. 9-13.

Lbova, L. V., Volkov, P. V., Kozhevnnikova, D. V. y Kulakobskaya, L. V. (2013), “Upper Paleolithic 'Sounding Artifacts' from Mesin, Ukraine: modification marks", Archaeology, Ethnology and Anthropology of Eurasia, pp. 22-32.

Levin, T., With Valentina Süzükei (2006), Where Rivers and Mountains sing. Sound, Music and Nomadism in Tuva and Beyond, Bloomington, Indianapolis, Indiana University Press.

Lewis, J. (2013), "A Cross-Cultural Perspective on the Significance of Music and Dance to Culture and Society: Insight from BaYaka Pygmies", M. A. Arbib (ed.), Language, Music, and the Brain, Cambridge (MA), The MIT Press, pp. 45-65

Li, L. (2000), "Ancestor worship: An archaeological investigation of ritual activities in Neolithic North China", Journal of East Asian Archaeology, 2, 1, pp. 129-164.

List, G. (1971), "On the Non-Universality of Musical Perspectives", Ethnomusicology, 15, 3, pp. 399-402.

- (1984), "Concerning the Concept of the Universal and Music", The World of Music, 26, 2, pp. 40-49.

Luca, S. (2014), Art and religious beliefs in the Neolithic and Aeneolithic from Romania, Sibiu, Ministeri Culturii. Muzeul National Brukenthal.

Lund, C. (1981), "The archaeomusicology of Scandinavia", World Archaeology: Archaeology and musical instruments, 12, 3, pp. 246-265.

- (2010), "Music Archaeology in Scandinavia", S. Mirelman (ed.), The Historiography of Music in Global Perspective, New Jersey, Gorgias Press, pp. 177-207.

Lund, C. y Larsson, G. (1974), Klang i Flinta och brons. Catálogo de exposición, Stockholm, Musikmuseet.

Macadam, R. (1860), "Ancient Irish Trumpets", Ulster Journal of Archaeology, 8, pp. 99-110.

Marin, C., Cristina, N. E., Teodor, O., Ovidiu, C., Ionuț, L. F. y Marian, L. (2019), "Wind instruments in the mid upper palaeolithic of the eastern carpathians", Annales d'Universite 'Valahia' Targoviste, Section d'Archeologie et d'Histoire, 1, 21, pp. 7-19.

Martí Oliver, B., Arias-Gago Del Molino, A., Martínez Valle, R. y Juan-Cabanilles, J. (2001), "Los tubos de hueso de la Cova de L'Or (Beniarrés, Alicante). Instrumentos musicales en el Neolítico Antiguo de la Península Ibérica", Trabajos de Prehistoria, 58, 2, pp. 4167.

Mattioli, T. y Díaz-Andreu, M. (2017), "Hearing rock art landscapes. A survey of the acoustical perception in the Sierra de San Serván area in Extremadura (Spain)", Time and Mind, 10, 1, pp. 81-96.

Mattioli, T., Farina, A., Armelloni, E., Hameau, P. y Díaz-Andreu, M. (2017), "Echoing landscapes: echolocation and the placement of rock art in the Central Mediterranean", Journal of Archaeological Science, 83, pp. 12-25.

Mazo Pérez, C., García Benito, C. y Alcolea Gracia, M. (2015), "Un caso de Arqueología Experimental aplicado a la Arqueología Musical", Salduie, 15, pp. 65-91. 
Megaw, J. V. S. (1984), "Problems and non-problems in palaeo-organology: a musical miscellany", J. M. Coles y D. D. A. Simpson (eds.), Studies in ancient Europe: Essays presented to Stuart Piggott, Leicester, Leicester University Press, pp. 333-358.

Merriam, A. P. (1964), The Anthropology of music, Evanston, Northwestern University Press. Münzel, S., Seeberger, F. y Hein, W. (2002), "The Geißenklösterle Flute - Discovery, Experiments, Reconstruction", E. Hickmann, A. Kilmer y R. Eichmann (eds.), Studien zur Musikarchäologie III, Radhen, Marie Leidorf, pp. 107-118.

Nettl, B. (2005), The study of ethnomusicology: thirty-one issues and concepts, Urbana, University of Illinois Press.

Normand, C., Goutas, N., Lacarrière, J. y Simonet, A. (2012), "El Gravetiense de la cueva de Isturitz: nuevas investigaciones, nuevos datos", C. de las Heras Martín, J. A. Lasheras Corruchaga, A. Arrizabalaga Valbuena y M. de la Rasilla Vives (eds.), Pensando el Gravetiense: nuevos datos para la región cantábrica en su contexto peninsular y pirenaico, Museo Nacional y Centro de Investigación de Altamira, pp. 161-183.

Olsen, D. A. (2001), Music of El Dorado The ethnomusicology of ancient south American cultures, Gainesville, University Press of Florida.

P. (1833), "Ancient Irish Trumpets", The Dublin Penny Journal, 2, 56, pp. 27.

Passemard, E. (1913), "Fouilles à Isturitz (Basses-Pyrénées)", Bulletin de la Société Préhistorique Française, pp. 647-649.

Peyrony, D. (1930), "Sur quelques pièces intéressantes de la grotte de La Roche, près de Lalinde (Dordogne)", L'Anthropologie, 40, pp. 19-29.

Piette, É. (1874a), "Une flûte néolithique", Comptes rendus de l'Académie des Sciences, 79, pp. 56-60.

- (1874b), "La flûte composée à l'Age du Renne", Comptes rendus de l'Académie des Sciences, 79, pp. 1277-1278.

Piggott, S. (1959), "The Carnyx in Early Iron Age Britain", The Antiquaries Journal, 39, 1-2, pp. 19-32.

Pomberger, B.-M., Kotova, N. y Stadler, P. (2018), "Flutes of the first European farmers", Ann. Naturhist. Mus. Wien, Seria A., 120, pp. 453-470.

Rainio, R. y Mannermaa, K. (2014), "Tracing the rattle of animal tooth pendants from the Middle Neolithic graves of Ajvide, Gotland, Sweden", World Archaeology, 46, 3, pp. 332-348.

Reynolds, P. J. (1979), Iron-age farm: the Butser experiment, London, British Museum Publications.

Reznikoff, I. (1987), "Sur la dimension sonore des grottes à peintures paléolithiques", Comptes rendus de l'Académie des Sciences, tome 304, série II, y tome 305, série II, Paris, pp. 153-156 y 307-310.

- (2008), "Sound resonance in prehistoric times: A study of Paleolithic painted caves and rocks", The Journal of the Acoustical Society of America, 123, 5, pp. 4138-4141.

(2012), "On the Sound Related to Painted Caves and Rocks", J. Ikäheimo, A.-K. Salmi y T. Älkä (eds.), Sounds Like Theory. XII Nordic Theoretical Archaeology Group Meeting in Oulu 25.-28.4.2012. Monographs of the Archaeological Society of Finland 2, Helsinki, The Archaeological Society of Finland, pp. 101-109.

Reznikoff, I. y Dauvois, M. (1988), "La dimension sonore des grottes ornées", Bulletin de la Société Préhistorique Française, 85, 8, pp. 238-246.

Rubio De Miguel, I. y Baena Preysler, J. (2002), "Instrumentos musicales en la Prehistoria: dificultades y vías de estudio", Patrimonio Musical, Granada, Centro de Documentación Musical de Andalucía, pp. 161-186.

Russell, N. (2005), "Çatalhöyük Worked Bone”, I. Hodder (ed.), Changing Materialities at 
Çatalhöyük: Reports from the 1995-99 Seasons, Ankara, British Institute, pp. 339-368.

Sabin, A. L., Papureanu, A.-M. y Florescu, C. T. (2018), "A possible musical instrument made from a fossil valve belonging to the family of Ostreidae (Rafinesque, 1815) from Tartaria Gura Luncii (Systematic Research Campaign 2018), Transylvania, Romania", Brvkenthal Acta Mvsei, Sibiu / Hermannstadt, Ministerul Culturii, pp. 35-62.

Salimpoor, V. N., Benovoy, M., Larcher, K., Dagher, A. y Zatorre, R. J. (2011), "Anatomically distinct dopamine release during anticipation and experience of peak emotion to music", Nature Neuroscience, 14, 2, pp. 257-262.

Scarre, C. y Lawson, G. (eds.), (2006), Archaeoacoustics, Cambridge, Cambridge University Press.

Seeger, A. (1987), Why Suyá sing: a musical anthropology of an Amazonian people, Cambridge, Cambridge University Press.

Shi-Yue, L. (1988), "Neolithic bone flutes from Hemudu, China", E. Hickmann y D. W. Hughes (eds.), The Archaeology of Early Music Cultures. Third International Meeting of the ICTM Study Group of Music Archaeology, Bonn, Verlang für systematische Musikwissenschaft GMBH, pp. 51-53.

Skeates, R. (1991), "Triton's trumpet: a Neolithic symbol in Italy", Oxford Journal of Archaeology, 10, 1, pp. 17-31.

Stone, W. H. (1878), "Experiments on Ancient Instruments", The Musical Times and Singing Class Circular, 19, 422, pp. 204-205.

Thomsen, C. J. (1848), Guide to Northern Archaeology by the Royal Society of Northern Antiquaries of Copenhagen (English translation), London, James Bain.

Tilley, C. Y. (1994), A phenomenology of landscape: places, paths and monuments, Oxford, Berg.

Trehub, S. E., Becker, J. y Morley, I. (2015), "Cross-cultural perspectives on music and musicality", Philosophical Transactions: Biological Sciences, 370, 1664, pp. 1-9.

Tsuge, G. I. (1988), "Stone and Clay Aerophone in Early Japan", E. Hickmann y D. W. Hughes (eds.), The Archaeology of Early Music Cultures. Third International Meeting of the ICTM Study Group of Music Archaeology, Bonn, Verlang für systematische Musikwissenschaft GMBH, pp. 89-96.

Tuniz, C., Bernardini, F., Turk, I., Dimkaroski, L., Mancini, L. y Dreossi, D. (2012), "Did Neanderthals play music? X-Ray computed micro-tomography of the Divje Babe 'flute"', Archaeometry, 54, 3, pp. 581-590.

Țurcanu, S. (2018), "The Birds in the Imaginarium of Cucuteni-Trypillia World. New Plastic Representations", S. Țurcanu y C.-E. Ursu (eds.), Materiality and Identity in Pre- and Protohistoric Europe. Homage to Cornelia-Magda Lazarovici. Suceava, The Bucovina Museum, pp. 297-343.

Turk, I. (ed.) (1997) Mousterian bone flute and other finds from Divje Babe I cave site in Slovenia, Ljubljana, Instituti Archaeologici Sloveniae.

Turk, M., Turk, I. y Otte, M. (2020), "The Neanderthal Musical Instrument from Divje Babe I Cave (Slovenia): A Critical Review of the Discussion", Applied Sciences, 10, 4, pp. 1226.

Villalba, M. J., Bañolas, L., Arenas, J. y Alonso, M. (1986), Les Mines neolítiques de Can Tintorer. Gavà. Excavacions 1978-1980, Barcelona, Generalitat de Catalunya

Vitezović, S. (2017), "Musical Instruments in the Central Balkan Neolithic", O. Tutilă, N. C. T. L. RişCuţA y I. V. Ferencz (eds.), Archaeological small finds and their significance. Proceedings of the Symposium Games and Toys, Cluj-Napoca, Editura Mega, pp. 7-15.

Von Hornbostel, E. M. y Sachs, C. (1961), "Classification of Musical Instruments: Translated 
from the Original German by Anthony Baines and Klaus P. Wachsmann", The Galpin Society Journal, 14, pp. 3-29.

Walker, J. C. (1786), Historical Memoirs of the Irish Bards. Interspersed with Anecdotes of the Music of Ireland. Also, an Historical Account of the Musical Instruments of the Ancient Irish. And an Appendix, containing Select Irish Melodies, London, Printed for T. Payne and Son.

Waller, S. J. (1993), "Sound reflection as an explanation for the content and context of rock art”, Rock Art Research, 10, 2, pp. 105-113.

- (1993), "Legends of Echoes Linked through Acoustics to Prehistoric Art", Journal of the Acoustical Society of America, 106, pp. 2227.

Watson, A. (2001), "The sounds of transformations: acoustics, monuments and ritual in the British Neolithic", N. Price (ed.), The Archaeology of Shamanism, Londres, Routledge, pp. 178-192.

Watson, A. y Keating, D. (1999), "Architecture and sound: an acoustic analysis of megalithic monuments in prehistoric Britain", Antiquity, 73, 280, pp. 325-336.

Wyatt, S. (2006), The Drums of the Southern TRB Culture. PhD, University of Edinburgh.

- (2010), "Psychopomp and Circumstance or Shamanism in Context", R. Eichmann, E. Hickmann y L. C. Koch (eds.), Studien zur Musikarchäologie VII, Radhen, Marie Leidorf, pp. 129-150.

- (2012), "Sound Production in Early Aerophones. Short Report on a Work in Progress", R. Eichmann, F. Jianjun y L.-C. Koch (eds.), Studien zur Musikarchäologie VIII, Radhen, M. Leidorf, pp. 393-398.

Xinghua, X. (2002), "On the Musical Civilisation of the Neolithic Age in China as Illustrated by the Ancient Bone-Flutes (from 9,000 to 7,800 years ago) unearthed at Jiahu, Henan Province", E. Hickmann, R. Eichmann y A. Kilmer (eds.), Studien zur Musikarchäologie III, Rahden, Marie Leidorf, pp. 161-167.

Zhang, J. y Cui, Q. (2013), "The Jiahu Site in the Huai River Area", A. P. Underhill (ed.), A Companion to Chinese Archaeology, Oxford, Blackwell, pp. 194-212.

Zhang, J., Harbottle, G., Wang, C. y Kong, Z. (1999), "Oldest playable musical instruments found at Jiahu early Neolithic site in China", Nature, 401, 6751, pp. 366-368.

Zhang, J., Xinghua, X. y Lee, Y. K. (2004), "The early development of music. Analysis of the Jiahu bone flutes", Antiquity, 78, 302, pp. 769-778.

Zhang, J. y Xinghua, X. (2002), "Music in the 7th Millenium B.C.: The Natural and Social Background of the Origin of the Jiahu Bone-Flutes, Central China", E. Hickmann, R. Eichmann y A. Kilmer (eds.), Studien zur Musikarchäologie III, Rahden, Marie Leidorf, pp. 147-153. 